\title{
Nanoscale Triboelectric-Effect-Enabled Energy Conversion for Sustainably Powering Portable Electronics
}

\author{
Sihong Wang, ${ }^{\dagger, \S}$ Long Lin, ${ }^{\dagger, \S}$ and Zhong Lin Wang ${ }^{\dagger, \dagger, *}$ \\ ${ }^{\dagger}$ School of Materials Science and Engineering, Georgia Institute of Technology, Atlanta, Georgia 30332-0245, United States \\ ${ }^{\ddagger}$ Beijing Institute of Nanoenergy and Nanosystems, Chinese Academy of Sciences, Beijing, China
}

Supporting Information

ABSTRACT: Harvesting energy from our living environment is an effective approach for sustainable, maintenance-free, and green power source for wireless, portable, or implanted electronics. Mechanical energy scavenging based on triboelectric effect has been proven to be simple, cost-effective, and robust. However, its output is still insufficient for sustainably driving electronic devices/systems. Here, we demonstrated a rationally designed arch-shaped triboelectric nanogenerator (TENG) by utilizing the contact electrification between a polymer thin film and a metal thin foil. The working mechanism of the TENG was studied by finite element simulation. The output voltage, current density, and energy volume density reached $230 \mathrm{~V}, 15.5 \mu \mathrm{A} / \mathrm{cm}^{2}$, and $128 \mathrm{~mW} / \mathrm{cm}^{3}$, respectively, and an energy conversion efficiency as high as $10-39 \%$ has been

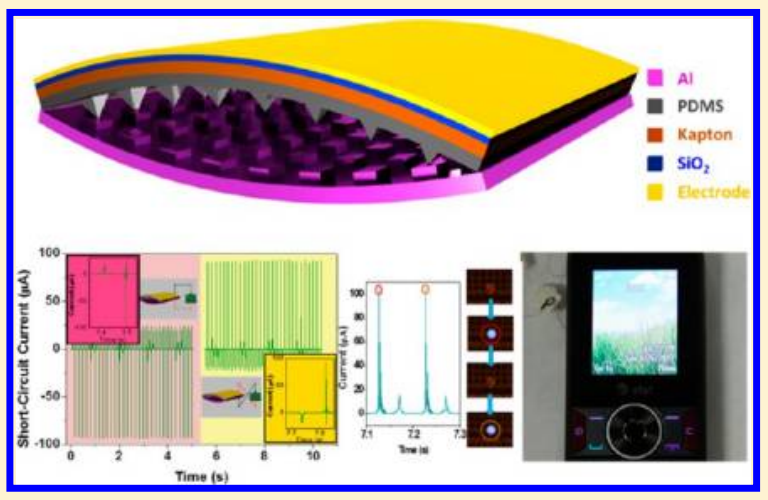
demonstrated. The TENG was systematically studied and demonstrated as a sustainable power source that can not only drive instantaneous operation of light-emitting diodes (LEDs) but also charge a lithium ion battery as a regulated power module for powering a wireless sensor system and a commercial cell phone, which is the first demonstration of the nanogenerator for driving personal mobile electronics, opening the chapter of impacting general people's life by nanogenerators.

KEYWORDS: Energy harvesting, triboelectric nanogenerator, self-powered system, lithium ion battery

\begin{abstract}
A rapid expansion of electronic devices ${ }^{1-4}$ toward wireless, portability, and multifunction desperately needs the development of independent and maintenance-free power sources. ${ }^{5-7}$ The emerging technologies for mechanical energy harvesting $^{8-10}$ are effective and promising approaches for building self-powered systems because of a great abundance of mechanical energy existing in our living environment and human body. Since 2006, piezoelectric nanogenerators $(\mathrm{PNGs})^{11-14}$ have been developed to efficiently convert tinyscale mechanical energy into electricity. Recently, another creative invention is the cost-effective and robust triboelectric nanogenerators (TENGs) ${ }^{15-17}$ based on the universally known contact electrification effect. ${ }^{18,19}$ TENG harvests mechanical energy through a periodic contact and separation of two polymer plates. However, in order to realize sustainable driving of electronic devices/systems, the output of TENG must be significantly improved through a rational design.

The two different types of nanogenerators presented above have a similar underlying physical process ${ }^{12,17}$ for producing electricity: generation of immobile charges (ionic charges for PNG or electrostatic charges on insulators for TENG), and a periodic separation and contact of the oppositely charged surfaces to change the induced potential across the electrodes, which will drive the flow of free electrons through an external load. The electrical output and efficiency are radically determined by the effectiveness of the above two processes.
\end{abstract}

As for the charge generation in TENG, maximizing the generation of electrostatic charges on opposite sides is critical, which can be achieved by selecting the materials with the largest difference in the ability to attract electrons ${ }^{20}$ and the modification of surface morphology. ${ }^{16,17}$ In another aspect, the effective periodic switching between separation and intimate contact of the two charged plates is vitally important to determine the electrostatic potential across the two electrodes, which is the driving force for the free electrons. For the platestructured TENG, it is not easy to achieve both complete contact and separation of the two oppositely charged plates upon pressing and releasing, especially under the electrostatic attraction between them. A firmly attached contact of the two oppositely charged surfaces is unfavorable for electricity generation. If we simply use a spacer between the two plates, ${ }^{17}$ it will then hinder the complete contact of the two plates. In this paper, we completely solved this problem through an archshaped TENG with a steady gap between the plates at strainfree conditions. This was achieved through ingeniously introducing surface thermal stress during thin film deposition. $^{21,22}$ On the basis of this unique structure, the voltage and current reached $230 \mathrm{~V}$ and $0.13 \mathrm{~mA}$ with an instantaneous

Received: September 25, 2012

Revised: November 1, 2012 


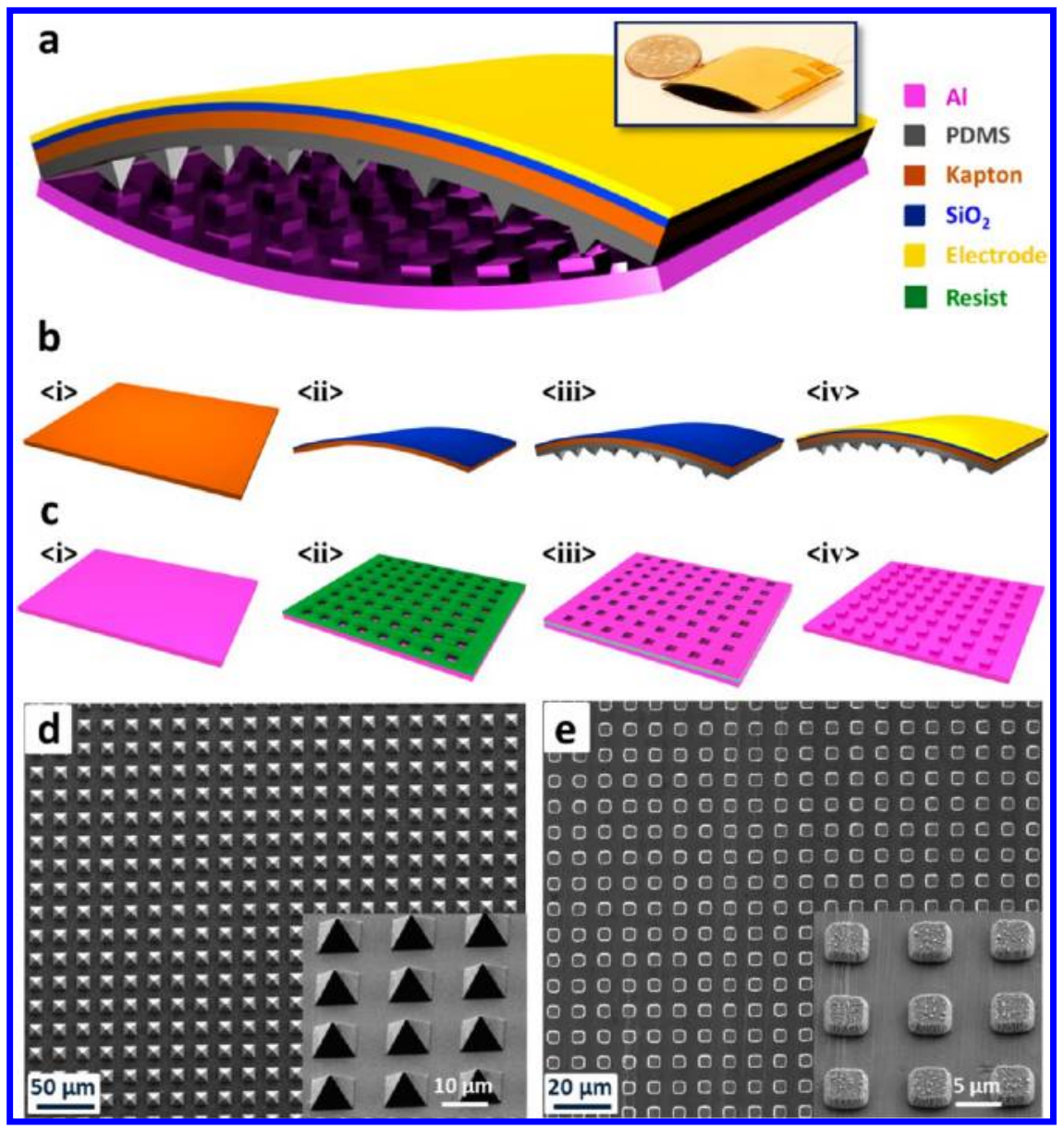

Figure 1. Structure and fabrication process of the arch-shaped triboelectric nanogenerator (TENG). (a) Schematic diagram showing the structural design of the arch-shaped TENG; the inset is the photograph of a typical arch-shaped TENG device. (b,c) Fabrication flowchart for (b) the top plate and (c) bottom plate of the TENG. (d,e) Top view SEM images of (d) the PDMS surface with pyramid patterns and (e) Al surface with cubic patterns; the insets are high-magnification images at a tilted angle.

maximum power density of $3.56 \mathrm{~mW} / \mathrm{cm}^{2}$ and $128 \mathrm{~mW} / \mathrm{cm}^{3}$ (note the latter was calculated using the entire volume of the device here and afterward unless specified). The power of a single device is high enough to continuously drive a LED. With the first realization of charging a lithium ion battery (LIB) to full capacity, the TENGs were combined with LIBs to form power modules for driving a wireless sensor system and a commercial cell phone. This is an unprecedented progress in rational optimization of the TENG design and its first application for self-powered systems, unambiguously demonstrating its feasibility for powering portable electronics, sensors for health care, environmental and infrastructure monitoring, and security.

The arch-shaped TENG is based on the contact electrification between patterned polydimethylsiloxane $(\mathrm{PDMS})^{16}$ as the top plate and patterned $\mathrm{Al}$ foil as the bottom plate (Figure 1a). According to the triboelectric series ${ }^{20,23}$ (Figure S1 in Supporting Information), the purposely chosen PDMS and $\mathrm{Al}$ are almost at the two ends with very large differences in ability to attract and retain electrons. The unique arch-shaped structure of the TENG from the naturally bent top plate, which helps to carry out the action of effective charge separation and contact using the elasticity of the film, is achieved by the following innovative fabrication process (Figure $1 b, c)$ : The top part starts from a piece of flat Kapton film (Figure $1 \mathrm{~b}<\mathrm{i}>$ ). A layer of $500 \mathrm{~nm} \mathrm{SiO}{ }_{2}$ film is deposited using plasma-enhanced chemical vapor deposition (PECVD) at 250 ${ }^{\circ} \mathrm{C}$ (Figure $1 \mathrm{~b}<\mathrm{ii}>$ ). Upon cooling to room temperature, the Kapton will shrink to a much larger extent than the $\mathrm{SiO}_{2}$ film because of the large difference in thermal expansion coefficients, so that thermal stress across the interface will make the plate bent naturally toward the $\mathrm{SiO}_{2}$ side (the curvature is calculated in supplementary discussion S1). Then, the prefabricated PDMS film with pyramid patterns (Figure S2) is glued to the inner surface through a thin PDMS bonding layer (Figure $1 \mathrm{~b}<\mathrm{iii}>$ ). Finally, the electrode is deposited on top (Figure $1 \mathrm{~b}<\mathrm{iv}>$ ). As for the bottom plate, an aluminum foil (Figure $1 \mathrm{c}<\mathrm{i}>$ ) is patterned with a typical photolithography process: defining the photoresist to the array of square windows (Figure 1c <ii $>$ ); depositing a layer of aluminum on top (Figure 1c <iii >); and finally lift-off, leaving the patterned $\mathrm{Al}$ cubes on the foil (Figure 1c $<$ iv $>$ ). At last, the two as-fabricated plates of the same size are attached face-to-face and sealed at the two ends. The soft $\mathrm{Al}$ plate will be forced to bend outward under the contraction from the other plate, so that a gap will form naturally between. The patterned surfaces of PDMS film (Figure 1d) and $\mathrm{Al}$ foil (Figure 1e) are fabricated to enhance the triboelectric charging and are characterized using scanning electron microscopy (SEM). Both arrays are uniform and regular across a very large area.

The operation of the arch-shaped TENG is realized by applying a cycled compressive force onto the whole area of the 


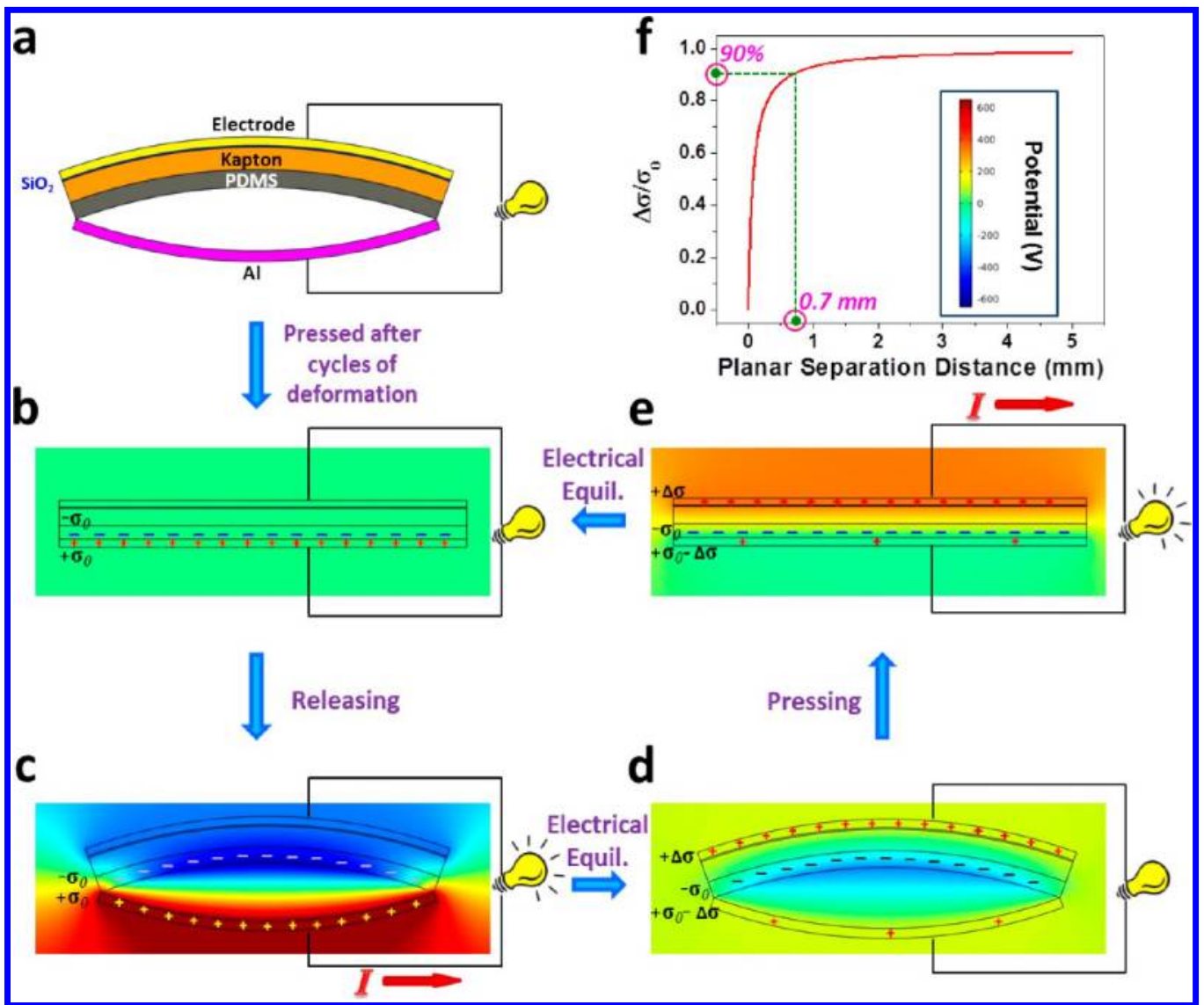

Figure 2. Working principle of the polymer-metal based TENG. (a) Two-dimensional sketch showing the initial state of the TENG before any deformation. $(\mathrm{b}-\mathrm{e})$ Finite element simulation of the periodic potential change between the two electrodes upon cyclic deformation, showing the driving force for the back-and-forth charge flow generated by the TENG. A cycle is generally divided into four states: (b) device under pressing, (c) deformation released, (d) charges transferred, and (e) device gets pressed again and final charge transferred again to cycle back to (b). (f) Relationship of the ratio between transferred charge density $(\Delta \sigma)$ and triboelectric charge density $\left(\sigma_{0}\right)$, with the planar separation distance, which shows the importance of the effective charge/plate separation. The inset is the scale of the voltage for the simulation results presented in (b-e).

device, so that the bending plates will be periodically pressed to flatten and contact closely with each other. Once released, the two plates will separate apart due to the stored elastic energy and revert instantaneously back to their original arch shape due to resilience. The working principle of the polymer-metal TENG is schematically depicted in Figure 2 using the numerically simulated electrostatic potential distribution arising from triboelectric charges (using COMSOL package). These semiquantitative results show that a cycled generation of the potential difference between the two electrodes drives the flow of electrons through an external load. At the original state before the contact of the triboelectric films (Figure 2a), there is no charge transferred, thus no electric potential. Upon the pressing of the two films toward each other, they will be brought into full surface contact and possibly relative sliding would occur, which results in electron transfer from a material in the positive side of the triboelectric series to the one in the negative side in the series (Figure S1). Accordingly, electrons will be injected from $\mathrm{Al}$ to the PDMS surface, leaving positive charges on the $\mathrm{Al}$ foil. Previous theoretical study on triboelectricity reveals that such a charge transfer process will continue in the first few hundreds of cycles until the accumulated charges reach a saturation and equilibrium; the negative charges will be preserved on the PDMS surface due to the nature of the insulator. ${ }^{24}$ However, the positive triboelectric charges on the conductive $\mathrm{Al}$ foil would attract the electrons in the opposite electrode to flow through an external load, which is the observed current in this case. This process is different from the mechanism proposed for the polymer-polymer based TENG. After cycles of deformation, when the device is pressed and the surfaces with charges are in close contact with each other, all of the triboelectric charges will stay on the inner surfaces with the same surface density $\left(\sigma_{0}\right)$ (Figure $2 \mathrm{~b}$ ). Thus, these charges with opposite signs will be virtually in the same plane, and there will be little potential difference across the two layers due to the negligible charge separation. Once the pressing force is released, the TENG will immediately rebound back to its original arch shape due to the elasticity of the film so that a gap will form again between the two plates. From the numerical simulation result, if the charge transfer has not happened at the moment, the electric field generated by the separated surface charges will give rise to a much higher potential on the Al foil side than the top electrode (TE) (Figure 2c). Such a potential difference will drive the flow of positive charges from $\mathrm{Al}$ foil to TE through the external load until the potential difference is fully offset by the transferred charges, rendering the TE with a surface charge density of $\Delta \sigma$, while the $\mathrm{Al}$ is left with $\sigma_{0}-\Delta \sigma$ (Figure $2 \mathrm{~d}$ ). Subsequently, when the TENG is pressed again to reach the close contact of the two plates, these redistributed charges will inversely build a positive potential on TE (Figure 2e), which will drive all of the transferred charges $(\Delta \sigma)$ to flow back to the inner surface of 


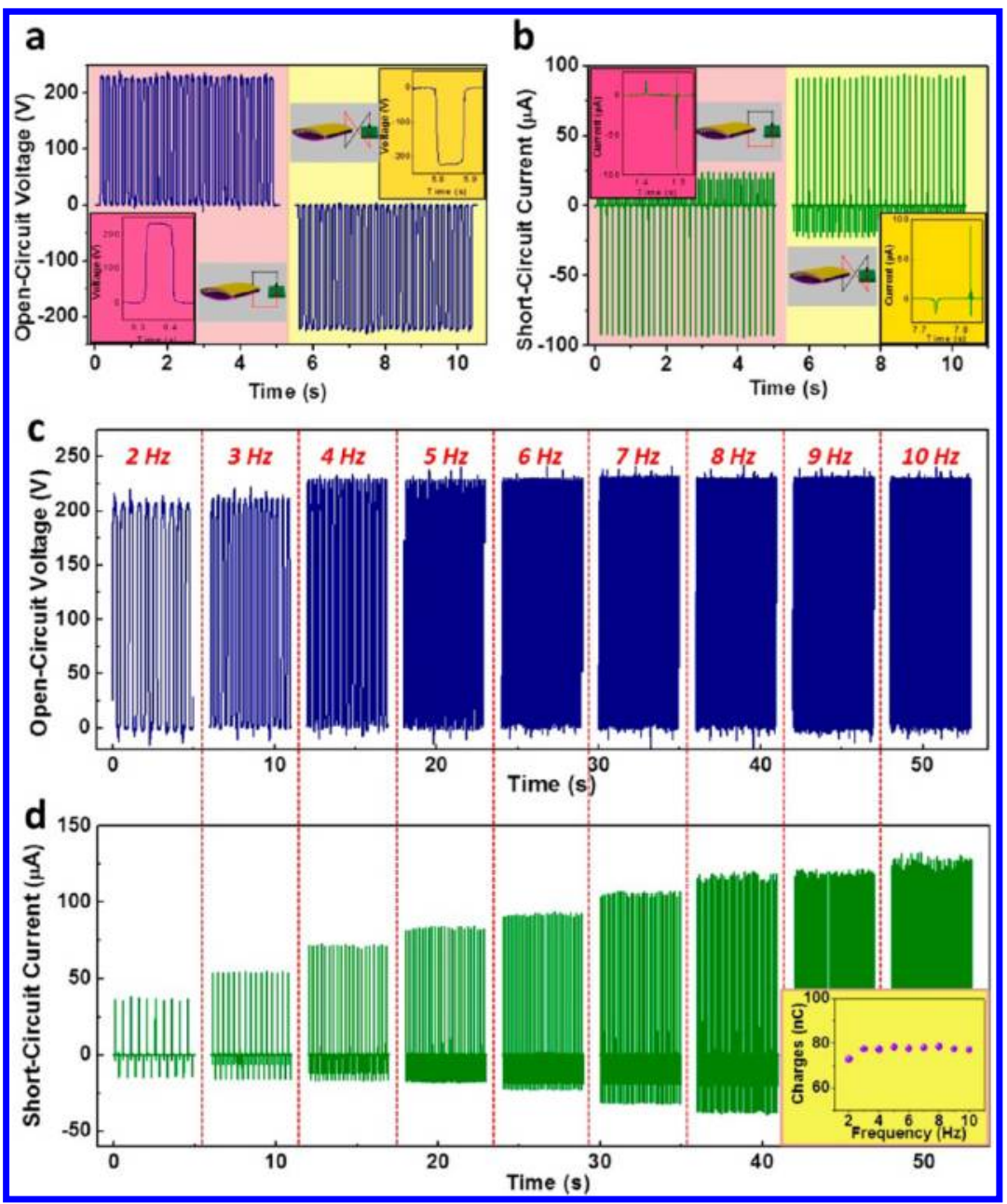

Figure 3. Characterizing the performance of the arch-shaped TENG. (a) Open-circuit voltage and (b) short-circuit current of the TENG under the deformation frequency of $6 \mathrm{~Hz}$. These are studies under both the forward connection (Al connected to the positive probe) shown at the left-hand side and reverse connection shown at the right-hand side. The insets are the magnified output curve in one cycle and the sketch of the corresponding connection polarity. (c,d) Influence of the deformation frequency on (c) the open-circuit voltage and (d) the short-circuit current. The inset in (d) is the integration of a single current peak from each frequency, which gives the total charges transferred in a half cycle, showing that the total contact charges generated are almost independent of the deformation frequency.

the $\mathrm{Al}$ foil. Then a cycle is achieved, and the device will go back to the equilibrium state depicted in Figure $2 \mathrm{~b}$. This is a full cycle of electricity generation.

From the above simulation result, we can easily tell that both the voltage and current outputs are related to the amount of charges transferred ( $A \Delta \sigma$, where $A$ is surface area of the plate), which is determined by the triboelectric charge density $\left(\sigma_{0}\right)$ and the separation distance of the two plates. As $\sigma_{0}$ is regarded as a constant, we did an analytical calculation based on the simplified model of quasi-infinite flat plates to give an idea on the magnitude of the distance required for the optimum output (supplementary discussion S2). The relationship of the ratio between $\Delta \sigma$ and $\sigma_{0}$, with the planar separation distance, is shown in Figure 2f. We can see that, when the separation distance starts to increase from 0 to $0.7 \mathrm{~mm}, \Delta \sigma$ keeps a very rapid increase from 0 to $\sim 90 \%$ of $\sigma_{0}$. Then, the slope of this curve starts to decrease. Thus, it can be concluded that both an intimate contact and a subsequent separation of nearly $1 \mathrm{~mm}$ are required for the phenomenal transferring of charges. This is just the unique innovation and advantage of the purposely designed arch-shaped structure, which introduces sufficient resilience for separating the plates but without sacrificing the intimate contact and electrification.

Under the above-described periodic deformation scenario, the electric output measurement was performed on an archshaped TENG device at a size of $3 \mathrm{~cm} \times 2.8 \mathrm{~cm}$, with the triggering frequency of $6 \mathrm{~Hz}$ and controlled amplitude. Since the accumulation of the triboelectric charges increases and reaches equilibrium in a certain period of time after multiple cycles, the output will gradually go up in the first stage upon deformation (Figure S3). Then, the open-circuit voltage $\left(V_{\mathrm{OC}}\right)$ will stabilize at $230 \mathrm{~V}$ (Figure 3a), measured by an electrometer with infinite input resistance. From the inset of Figure 3a, when the bottom $\mathrm{Al}$ is connected to the positive probe of the electrometer, upon the release of the pressing force, a positive voltage is generated because of the immediate charge separation. Since in an open-circuit condition the electrons cannot flow to screen the induced potential difference between the two electrodes, the voltage will hold at a plateau until the subsequent pressing deformation in the second half cycle. As shown in Figure 3b, the peak value of the short-circuit current $\left(I_{\mathrm{SC}}\right)$ reaches $94 \mu \mathrm{A}$, corresponding to the half cycle of pressing that is at a higher straining rate than releasing. The integration 


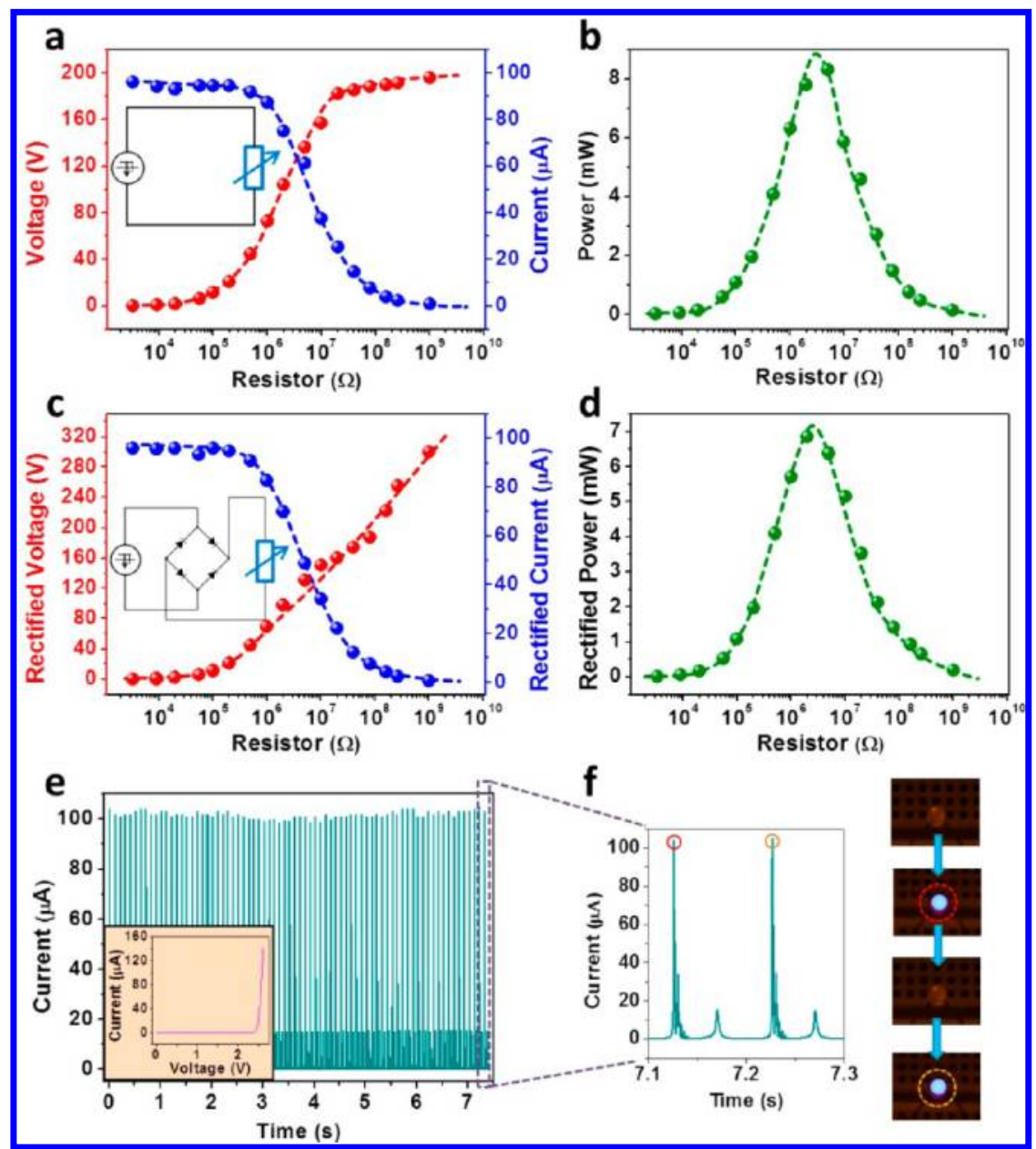

Figure 4. Arch-shaped TENG as a direct power source to drive electronic devices. (a,b) When the TENG driving a load without rectification, the dependence of (a) the output voltage, current, and (b) instantaneous power on the load resistance. (c,d) When the TENG driving a load with rectification, the dependence of (c) the output voltage, current, and (d) instantaneous power on the resistance of the load. All of the dots are measured values, and the solid lines are fitted curves. (e) Current through a LED driven by a rectified TENG under $8 \mathrm{~Hz}$. The inset is the characteristic $I-V$ curve of the LED. (f) Snapshots of the TENG-driven flashing LED, corresponding to the magnified current peaks.

of each current peak gives the total charges transferred in a half cycle of deformation. Moreover, when the connection polarity to the electrometer is switched, both the voltage and current signal will be completely reversed. The gap from the archshaped structure is a key factor for the enhanced output because, without the $\mathrm{SiO}_{2}$-film-introduced bending, there will be much smaller and unstable electrical output (Figure S4).

Besides the triboelectric charge density and the separation distance of the two plates, another factor that could influence the output of the TENG is the deformation frequency, which affects the straining rate. Since the mechanical energy from the environment is always irregular and varies in frequencies, it is necessary to study the dependence of TENG's output on the frequency. Thus, we tested the TENG device in a series of nine different frequencies, from 2 to $10 \mathrm{~Hz}$, with the amplitude of the triggering motor remaining constant. As shown in Figure $3 c, V_{\mathrm{OC}}$ almost remains the same at different frequencies. The probable reason is that, at the open-circuit condition, it does not involve the dynamic process of charge transfer. The voltage is only determined by the triboelectric charge density and the plate separation at any given time. But for the short-circuit current, it presents a very clear increasing trend with the increase of frequency, from $35 \mu \mathrm{A}$ at $2 \mathrm{~Hz}$ to $130 \mu \mathrm{A}$ at $10 \mathrm{~Hz}$ (Figure 3d), because the deformation rate increases with deformation frequency, which leads to a higher flow rate of charges, that is, higher current (Figure S5), but the total amount of charges transferred is constant at given triboelectric condition and separation distance. This is confirmed by the integration of every single current peak from each of the nine different frequencies (inset of Figure 3d). Therefore, the instantaneous power output increases with the increase of frequency, so that it will be more eligible to drive electronic devices with larger power consumption. From the above results, when the frequency reaches $10 \mathrm{~Hz}$, the instantaneous power output reaches up to $3.56 \mathrm{~mW} / \mathrm{cm}^{2}$ and $128 \mathrm{~mW} / \mathrm{cm}^{3}$.

The goal of the development of nanogenerators is to drive electronic devices by harvesting small-scale mechanical energy, so that the self-powered system can be realized. As for the energy conversion devices such as TENGs, there are generally two methodologies for them to be used as a power source. The first choice is to power devices directly using the pulses generated by TENGs, such as a chemical sensor ${ }^{25}$ and a LCD. ${ }^{26}$ In practice, the output power to the load depends on the match between the load and the power source. When the working TENG (under a frequency of $6 \mathrm{~Hz}$ ) is directly connected to the loads of different resistances, we can find that the current through the load will generally decrease from $I_{\mathrm{SC}}$ when the resistance increases from 0 (Figure $4 \mathrm{a}$ ), but the voltage across 


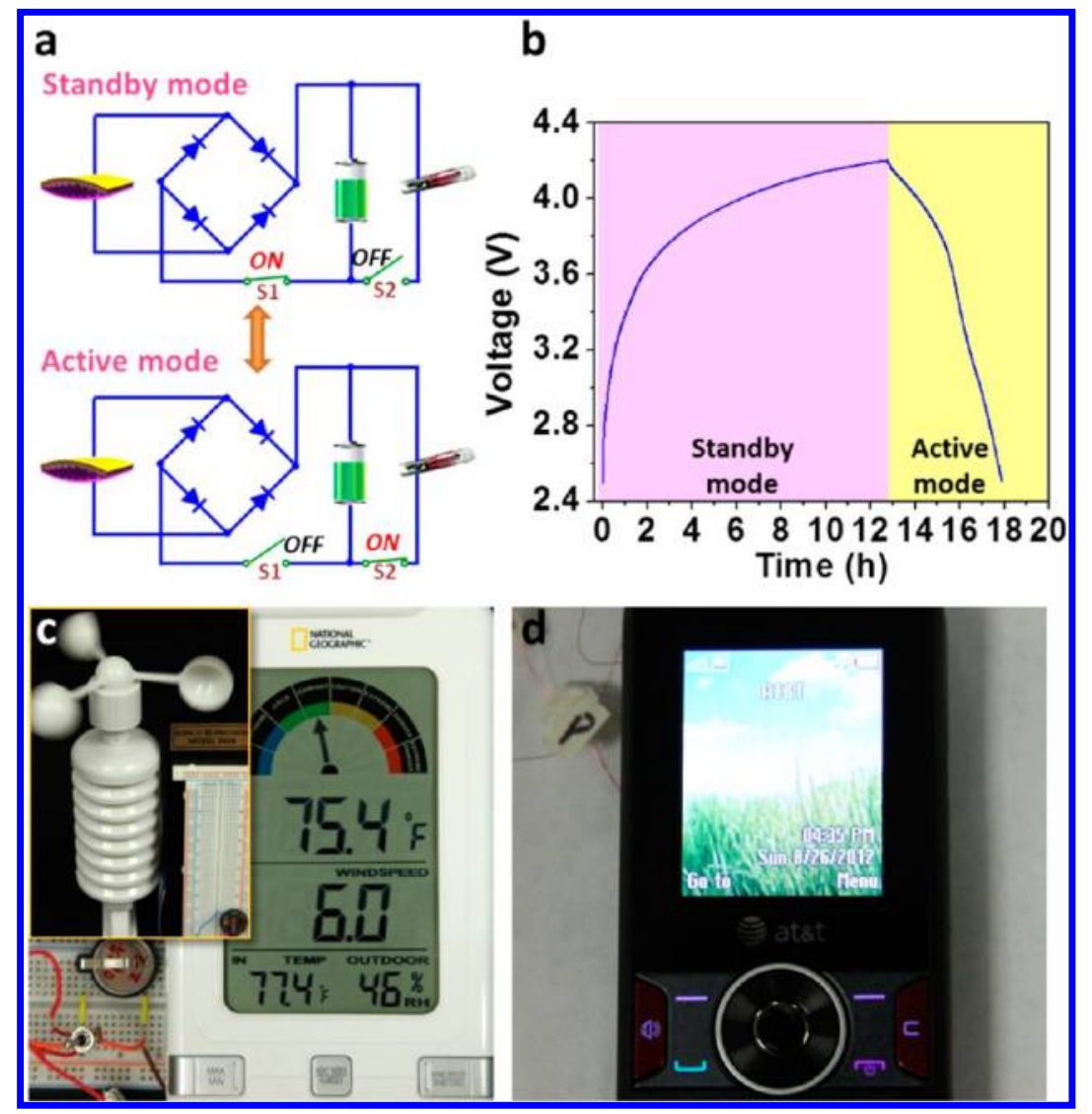

Figure 5. Power source module composed of an arch-shaped TENG and a lithium ion battery for driving portable electronics that need a regulated input power. (a) Schematic circuit diagram showing the switching between the "standby mode" and the "active mode" of the power source module. (b) Voltage curve showing the entire cycle of a lithium ion battery fully charged by TENG (standby mode), with the subsequent constant current discharge at $2 \mu \mathrm{A}$ (active mode). (c) Wireless wind and humidex thermometer, as an example of a whole wireless sensor network system, is driven by two such power source systems (one for the remote sensor module and one for the receiver-display module). (d) Commercial cell phone powered by its original battery that was charged by the TENG.

the load will follow an opposite trend and saturate to $V_{\mathrm{OC}}$ when the resistance gets infinitely large. The instantaneous power on the load will reach the maximum value of $9 \mathrm{~mW}$ at a load resistance of $\sim 3 \mathrm{M} \Omega$ (Figure $4 \mathrm{~b}$ ). On the basis of the above results, we have estimated the energy conversion efficiency for this structure. Since the thickness of the substrate used here has little influence on the electricity generation process but does consume a lot of mechanical energy, the output efficiency can be dramatically high if the thickness of the plate is reduced according to the feasible engineering fabrication while still maintaining the mechanical integrity of the TENG. On the basis of experimentally achievable design and fabrication, the efficiency of this structure with the same gap distance could be as high as $10-39 \%$ (supplementary discussion S3).

In the other case, when the TENGs are used to drive polar devices such as diodes, the AC signals of the NG need to be rectified before being connected to the load. We can find that both the rectified voltage and current follow a similar trend (Figure 4c) with the scenario of no rectification, and the only difference is that the voltage will not saturate but continues to increase as the load resistance above $100 \mathrm{M} \Omega$. The maximum output power is $7.2 \mathrm{~mW}$ at a load of $\sim 2 \mathrm{M} \Omega$ (Figure $4 \mathrm{~d}$ ), which is a bit smaller than the maximum power of $9 \mathrm{~mW}$ without rectification, simply because of the energy loss of the rectifier unit. The characteristic $I-V$ curves for these two scenarios are depicted in Figure S6.
The instantaneous output of the TENG can continuously drive LEDs. The real-time current signals flowing through the LED are given in Figure 4e. In comparison with the characteristic $I-V$ curve of the LED, the peak current of 100 $\mu \mathrm{A}$ is more than sufficient to turn the LED on. Figure $4 \mathrm{f}$ shows snapshots taken from a flashing LED. Each instant of lightingup corresponds to a large peak of the output current. In coordination with the TENG's output characteristics discussed above, the resistance of the LED in "on" state is only about 23 $\mathrm{k} \Omega$, far below the range ( 1 to $100 \mathrm{M} \Omega$ ) of rapid change of the current, so the current is almost the same as $I_{\mathrm{SC}}$, but the voltage on the LED is only $\sim 2.5 \mathrm{~V}$. From Figure $4 \mathrm{~b}$, because the resistance of such a single LED is far below the maximum power point, TENG can also drive the flashing of up to 50 LEDs connected in series (Figure S7 and videos S1 and S2), which only results in less than $5 \%$ decrease of the current. This demonstration clearly illustrates the ability of the TENG as a direct power source to continuously drive regular electronic devices.

In many cases, the AC pulses from the nanogenerators may not directly satisfy the need of some electronic devices/systems, because of either the requirement for a constant DC current or the higher power consumption than that generated by a single device. Thus, the output power needs to be regulated with an energy storage unit and a rectifier. ${ }^{27}$ As for the energy storage device, lithium ion batteries (LIBs) are the most widely used and promising choice. ${ }^{28}$ As the output (especially the current) 
from the arch-shaped TENG has reached the level to match the capacity of a LIB and overcome the self-discharge current, it will be feasible to use a TENG to charge a LIB. The electrical circuit of such a power source module is shown in Figure 5a. At the standby mode, the LIB is continuously charged by a TENG through a rectifier and is disconnected from the load (switch S1 on; S2 off). When enough charges have been stored, the module will move on to the active mode, in which the battery will be disconnected from the TENG charger ( $\mathrm{S} 1$ off) and start to power the system ( $\mathrm{S} 2$ on). Such a power source module is demonstrated using a homemade $\mathrm{LIB}$, with $\mathrm{LiCoO}_{2}$ as the cathode and graphite as the anode, with the same structure as commercial LIBs. In the standby mode, such a battery was fully charged by a TENG in $12.8 \mathrm{~h}$, with the battery voltage increased from 2.5 to $4.2 \mathrm{~V}$ (Figure $5 \mathrm{~b}$ ), which is the standard charging window for a commercial LIB of this type. The charging current provided by the TENG is shown in Figure S8. This is the first time a LIB is fully charged by a nanogenerator! Then in the "active mode", it can run for $5.2 \mathrm{~h}$ with a constant discharge current at $2 \mu \mathrm{A}$, corresponding to a total capacity of $10.4 \mu \mathrm{Ah}$.

Such an operation model of switching between the two modes is suitable for a lot of applications, such as wireless sensor network systems that have a long standby mode and a short active mode. ${ }^{29}$ The schematic diagram of such a system is shown in Figure S9. If each module can be driven by an independent power source composed of a TENG and a LIB, the operation of the wireless sensor network could be maintenance-free. We demonstrated the powering of a wireless wind and humidex thermometer (Figure 5c and video S3), which consists of two parts-an outdoor remote sensor panel to measure the temperature, humidity, and the wind speed, as well as to transmit the data back to the indoor receiver; a display panel with a data receiver, a data processing unit, and an integrated temperature sensor. We use two TENG-charged batteries (with charging curves similar to that in Figure $5 b$ ) to power these two components (located $\sim 10 \mathrm{~m}$ apart), respectively, and a self-powered wireless sensor network system is realized. This type of power source module could solve two vital limitations for a battery: ${ }^{30}$ (1) the periodical replacement of a drained battery; (2) the relatively big size and weight of the battery to ensure a longer life operation. Thus, this will realize the miniaturization of a battery or extend its lifetime, especially for nanosystems because they can be charged up at any time. The TENG can also be used together with a capacitor, in the case of fast charging and discharging, since it can charge a $47 \mu \mathrm{F}$ capacitor to $10 \mathrm{~V}$ in $8 \mathrm{~min}$ (Figure $\mathrm{S} 10$ ).

Another huge application of the combination of nanogenerator with battery could be in people's daily life, to power personal electronics. With the greatly enhanced output from a 9 $\mathrm{cm}^{2}$ size single-layer arch-shaped TENG, we successfully used it to charge a commercial cell phone battery (which was completed drained, Figure S11). Then, it could drive a cell phone up again, including making a phone call (Figure $5 \mathrm{~d}$ and video S4). Since the current of the TENG should be proportional to its area, if the TENG is scaled up in three dimensions, the charging rate could be tens of times higher, which is very feasible to fully charge a cell phone battery.

In summary, by rational design of an arch-shaped structure based on the contact electrification between a polymer and a metal film, we demonstrated a novel type of triboelectric nanogenerator with much improved power output of 3.56 $\mathrm{mW} / \mathrm{cm}^{2}$ and $128 \mathrm{~mW} / \mathrm{cm}^{3}$ (voltage of $230 \mathrm{~V}$ and current of
$0.13 \mathrm{~mA}$ ); and an estimated conversion efficiency of as high as $10-39 \%$ is possibly achievable. Such high output is achieved through controllable and revolutionary improvement on the effectiveness of charge separation and recontact process by purposely introducing thermal stress in the device fabrication. The mechanism for the first polymer-metal based TENG is illustrated through numerical simulation. The application of using the TENG as a power source has been demonstrated for two scenarios. TENG has been used to directly drive the continuous operation of an LED without rectification or storage. TENG has also been used to build up a power module together with a lithium ion battery for driving a wireless sensor system as well as a commercial cell phone. This study demonstrates a ground-breaking progress in development of high-power triboelectric nanogenerators, with outstanding applications as an effective power source for self-powered systems in sensor networks, portable electronics, biosensing and health care, environmental and infrastructure monitoring, and security.

Methods. Fabrication of the PDMS Film with Pyramid Patterns. The fabrication process starts from photolithographic patterning of 4 in. (100) $\mathrm{Si}$ wafers with thermally grown $\mathrm{SiO}_{2}$ on top. The patterned wafer with the array of square window openings was first etched by a buffered-oxide etching process to transfer the pattern onto the $\mathrm{SiO}_{2}$ layer. Then, the wafer was etched in $\mathrm{KOH}$ solution to fabricate the recessed features of a pyramid. After being cleaned with acetone, isopropyl alcohol, and ethanol in sequence, the $\mathrm{Si}$ molds were treated with trimethylchlorosilane (Sigma Aldrich) by gas phase silanization to enable the easy peel-off of the PDMS film from the Si mold in the following step. In preparing the patterned PDMS film, the elastomer and the cross-linker (Sylgard 184, Dow Corning) were mixed in a 10:1 ratio $(\mathrm{w} / \mathrm{w})$ and then casted on the Si mold. After the degassing process under vacuum, the mixture was spin-coated on the Si mold at $500 \mathrm{rpm}$ for $60 \mathrm{~s}$. After the thermal curing process at $85{ }^{\circ} \mathrm{C}$ for $1 \mathrm{~h}$, the PDMS inked with pyramid patterns was peeled off from $\mathrm{Si}$ mold. The surface without patterns will be glued to the inner surface of the bending Kapton substrate.

Assembly of the Lithium Ion Battery. First, two different slurries for the two working electrodes were made, which contain 70 wt $\%$ active material $\left(\mathrm{LiCoO}_{2}\right.$ for cathode and graphite for anode), $10 \mathrm{wt} \%$ carbon black powders as conductor, 20 wt \% polyvinylidene fluoride (PVDF) binder, and $\mathrm{N}$-methyl-2-pyrrolidone (Sigma Aldrich) as the solvent. Then the slurries were casted onto the current collectors ( $\mathrm{Al}$ foil for the cathode and $\mathrm{Cu}$ foil for the anode) with a uniform thickness of $10 \mu \mathrm{m}$. The electrodes were baked at $110{ }^{\circ} \mathrm{C}$ under vacuum for $12 \mathrm{~h}$. Then, 2016 stainless-steel coin cells were used for the battery assembly. The cathode and anode electrodes were stacked with a piece of polyethylene (PE) separator (MTI Corporation) between. After the system was filled with electrolyte (1 M LiPF6 in 1:1:1 ethylene carbonate/dimethyl carbonate/diethyl carbonate, Novolyte Technologies), the coin-cell was finally sealed.

\section{ASSOCIATED CONTENT}

\section{S Supporting Information}

Additional discussions, figures, and videos. This material is available free of charge via the Internet at http://pubs.acs.org. 


\section{AUTHOR INFORMATION}

\section{Corresponding Author}

*E-mail: zlwang@gatech.edu.

\section{Author Contributions}

$\S$ These authors contributed equally.

\section{Notes}

The authors declare no competing financial interest.

\section{ACKNOWLEDGMENTS}

Research was supported by DARPA (HR0011-09-C-0142), Airforce, U.S. Department of Energy, Office of Basic Energy Sciences under Award DEFG02- 07ER46394, NSF (CMMI 0403671), and the Knowledge Innovation Program of the Chinese Academy of Sciences (Grant KJCX2-YW-M13).

\section{REFERENCES}

(1) Tian, B. Z.; Cohen-Karni, T.; Qing, Q.; Duan, X. J.; Xie, P.; Lieber, C. M. Science 2010, 329, 830-834.

(2) Patolsky, F.; Timko, B. P.; Yu, G. H.; Fang, Y.; Greytak, A. B.; Zheng, G. F.; Lieber, C. M. Science 2006, 313, 1100-1104.

(3) Avouris, P.; Martel, R. MRS Bull. 2010, 35, 306-313.

(4) Dionne, J. A.; Sweatlock, L. A.; Sheldon, M. T.; Alivisatos, A. P.; Atwater, H. A. IEEE J. Sel. Top. Quantum Electron. 2010, 16, 295-306.

(5) Wang, Z. L. Adv. Mater. 2012, 24, 280-285.

(6) Paradiso, J. A.; Starner, T. IEEE Pervasive Comput. 2005, 4, 1827.

(7) Arico, A. S.; Bruce, P.; Scrosati, B.; Tarascon, J. M.; Van Schalkwijk, W. Nat. Mater. 2005, 4, 366-377.

(8) Mitcheson, P. D.; Miao, P.; Stark, B. H.; Yeatman, E. M.; Holmes, A. S.; Green, T. C. Sens. Actuators, A 2004, 115, 523-529.

(9) Beeby, S. P.; Torah, R. N.; Tudor, M. J.; Glynne-Jones, P.; O’Donnell, T.; Saha, C. R.; Roy, S. J. Micromech. Microeng. 2007, 17, $1257-1265$.

(10) Wang, Z. L.; Song, J. H. Science 2006, 312, 242-246.

(11) Wang, X. D.; Song, J. H.; Liu, J.; Wang, Z. L. Science 2007, 316, 102-105.

(12) Yang, R. S.; Qin, Y.; Dai, L. M.; Wang, Z. L. Nat. Nanotechnol. 2009, 4, 34-39.

(13) Chang, C. E.; Tran, V. H.; Wang, J. B.; Fuh, Y. K.; Lin, L. W. Nano Lett. 2010, 10, 726-731.

(14) Xu, S.; Hansen, B. J.; Wang, Z. L. Nat. Commun. 2010, 1.

(15) Fan, F. R.; Tian, Z. Q.; Wang, Z. L. Nano Energy 2012, 1, 328334.

(16) Fan, F. R.; Lin, L.; Zhu, G.; Wu, W. Z.; Zhang, R.; Wang, Z. L. Nano Lett. 2012, 12, 3109-3114.

(17) Zhu, G.; Pan, C. F.; Guo, W. X.; Chen, C. Y.; Zhou, Y. S.; Yu, R. M.; Wang, Z. L. Nano Lett. 2012, 12, 4960-4965.

(18) Lowell, J.; Roseinnes, A. C. Adv. Phys. 1980, 29, 947-1023.

(19) Castle, G. S. P. J. Electrost. 1997, 40-1, 13-20.

(20) Cross, J. A. Electrostatics: Principles, Problems and Applications; Hilger: Bristol, UK, 1987; p xii.

(21) Hsueh, C. H. J. Appl. Phys. 2002, 91, 9652-9656.

(22) Sinha, A. K.; Levinstein, H. J.; Smith, T. E. J. Appl. Phys. 1978, 49, 2423-2426.

(23) Diaz, A. F.; Felix-Navarro, R. M. J. Electrost. 2004, 62, 277-290.

(24) Saurenbach, F.; Wollmann, D.; Terris, B. D.; Diaz, A. F. Langmuir 1992, 8, 1199-1203.

(25) Xu, S.; Qin, Y.; Xu, C.; Wei, Y. G.; Yang, R. S.; Wang, Z. L. Nat. Nanotechnol. 2010, 5, 366-373.

(26) Hu, Y. F.; Zhang, Y.; Xu, C.; Zhu, G. A.; Wang, Z. L. Nano Lett. 2010, 10, 5025-5031.

(27) Hu, Y. F.; Zhang, Y.; Xu, C.; Lin, L.; Snyder, R. L.; Wang, Z. L. Nano Lett. 2011, 11, 2572-2577.

(28) Tarascon, J. M.; Armand, M. Nature 2001, 414, 359-367.

(29) Yick, J.; Mukherjee, B.; Ghosal, D. Comput. Networks 2008, 52, 2292-2330.
(30) Anastasi, G.; Marco, C.; Di Francesco, M.; Passarella, A. Ad Hoc Networks 2009, 7, 537-568. 